\title{
Extraocular Muscle Paresis, CTCAE
}

National Cancer Institute

\section{Source}

National Cancer Institute. Extraocular Muscle Paresis, CT CAE. NCI Thesaurus. Code C143466.

A disorder characterized by incomplete paralysis of an extraocular muscle. 